\section{Christoph Bachmann}

\section{Einleitung}

Astaxanthin (Abb. 1) ist ein roter Farbstoff aus der Familie der Carotinoide (wie unter anderem auch das bekannte Beta-Carotin). Es kommt weit verbreitet in Meerestieren vor; darin inbegriffen sind Schalentiere wie Garnelen, Krabben und Fische wie Lachs und Brachsen. Astaxanthin wird schon seit langer Zeit aus Hummer isoliert.

Zuerst wurde der Farbstoff nur in der Aquakultur zur Pigmentierung verwendet. 1991 wurde erstmals über die potenten antioxidativen Eigenschaften der Carotinoide berichtet [2]. Damit begann die Nachfrage nach Astaxanthin als Nahrungsergänzungsmittel. Heutzutage wird immer mehr Forschung betrieben, und die Nachfrage nach natürlichem Astaxanthin als Anwendung für die menschliche Gesundheit wächst explosionsartig.

Im Folgenden werden die Grundlage der Bioaktivität und die gesundheitsfördernden Wirkungen von Astaxanthin, das aus der Mikroalge Haematococcus pluvialis gewonnen wird, vorgestellt. Weiter werden die praktischen medizinischen Anwendungen mit Fallberichten aufgezeigt.

\section{Antioxidative Eigenschaften}

Die antioxidativen Eigenschaften von Astaxanthin beruhen auf drei Prinzipien:

(1) Die inhibitorische Aktivität von Astaxanthin auf die vom PeroxidRadikal gesteuerte Lipidperoxidation von homogenisierten Rattenmitochondrien erweist sich 100-mal grösser als die von Alpha-Tocopherol [2]. Verglichen mit 27 bekannten

\title{
Astaxanthin - ein medizinisches Nahrungsmittel
}

Eine 2013 veröffentlichte Studie [1] befasste sich mit den Eigenschaften von Astaxanthin, einem roten Farbstoff aus der Familie der Carotinoide. Die antioxidativen Eigenschaften sowie die allfälligen medizinischen Möglichkeiten der vor allem in Meerestieren vorkommenden Substanz werden in dieser Publikation vorgestellt. Der folgende Beitrag stellt eine gekürzte Fassung dieses Artikels dar.

Abb. 1. Chemische Struktur von Astaxanthin.

hydrophilen und lipophilen Antioxidantien besitzt Astaxanthin die stärkste Wirkung gegen den sehr reaktiven Singulett-Sauerstoff. Die Hydroxyl-Radikal-hemmende Eigenschaft von in Liposomen verkapseltem Astaxanthin ist stärker als jene von Alpha-Tocopherol [3].

(2) Im Gegensatz zu anderen Carotinoiden wie z.B. Beta-Carotin oder Lycopen besitzt Astaxanthin ausschliesslich antioxidative, keinesfalls aber auch prooxidative Eigenschaften [4]. Die Photostabilität von drei Carotinoiden in humanen Hautfibroblasten war Astaxanthin > Canthaxanthin > Beta-Carotin. Lediglich Astaxanthin vermag die apoptotische Wirkung von UVA-Strahlen ausser Kraft zu setzen [5].

(3) Astaxanthin fängt Radikale nicht nur an der konjugierten Polyenkette, sondern auch am terminalen Ring, in dem der Wasserstoff an Position 3 des Methins das Radikal-bindende Element zu sein scheint. Aufgrund seiner chemischen Eigenschaften scheint Astaxanthin in der Lage zu sein, Radikale sowohl an der Oberfläche als auch im Inneren der Phospholipidmembran zu beseitigen, obwohl seine ungesättigte Polyenkette Radikale nur in der Membran erfasst.

\section{Gesundheitsfördernde Eigenschaften}

Yuan et al. [6] haben in einem Übersichtsartikel potenzielle gesundheitsfördernde Wirkungen als Prävention und Behandlung von verschiedenen Krankheiten vorgestellt - wie Krebs, chronisch-entzündliche Erkrankungen, metabolisches Syndrom, Diabetes, diabetische Nephropathie, kardiovaskuläre Erkrankungen, Lebererkrankungen, neurodegenerative Erkrankungen, Augenerkrankungen, Hauterkrankungen, trainingsbedingte Müdigkeit sowie männliche Infertilität. Die genannten Anwendungsmöglichkeiten wurden in 65 klinischen Studien überprüft, deren Ergebnisse in mehr als 300 Peer-reviewed-Artikeln publiziert worden sind [710]. Diese präventiven und therapeutischen Möglichkeiten von Astaxanthin beruhen auf folgenden Wirkungen:

- Neurovaskuläre Protektion: Verminderung der Erythrozyten-Oxidation.

- Auge: Vermindert Augenmüdigkeit bei intensiver Computerarbeit.

- Haut: Präventive Wirkung gegen UV-bedingte Hautfalten. Verbesserung der Elastizität und Hauttrockenheit.

\section{KARGER}

(C) 2015 S. Karger GmbH, Freiburg

Fax +497614520714 
- Immunsystem: Verminderung von DNA-Schäden in Immunzellen und Verbesserung der Immunantwort.

- Metabolismus: Verbesserung der Insulin-Glukose-Balance, der Insulinresistenz und -sensibilität, Verminderung inflammatorischer Marker und der Progression von Leberverfettung sowie Verbesserung der Pankreasfunktion.

- Herz-Kreislauf: Verbesserung des Blutdrucks. Prävention von Lipidperoxidation und vaskulärer Entzündung.

- Gastrointestinaltrakt: Verminderung von Entzündungen mit Helicobacter pylori, Ulzera, Reflux, Gastritis und anderen Verdauungsstörungen.

\section{Literatur}

1 Yamashita E: Astaxanthin as a medical food. Funct Foods Health Disease 2013;3:254-258.

2 Miki W: Biological functions and activities of animal carotenoids. Pure Appl Chem 1991;63: 141-146.

3 Hama S, Uenishi S, Yamada A, et al: Scavenging of hydroxyl radicals in aqueous solution by astaxanthin encapsulated in liposomes. Biol Pharm Bull 2012;35:2238-2242.

4 Martin HD, Ruck C, Schmidt M, et al: Chemistry of carotenoid oxidation and free radical reactions. Pure Appl Chem 1999;71:2253-2262.
- Skelettmuskeln: Verbesserung der Ausdauer und Regeneration nach dem Training, Verminderung von Muskelschädigung und -atrophie.

- Kapillarzirkulation: Verbesserung der Zirkulation und Kapillarstabilität sowie Verminderung von BlutzellOxidation und Thromboserisiko.

- Fertilität: Verbesserung der Spermienparameter und der Fertilität.

\section{Fazit}

Beim Carotinoid-Farbstoff Astaxanthin handelt es sich um ein sehr wirksa- mes Antioxidans, dessen Wirksamkeit die antioxidativen Eigenschaften vieler bekannter Substanzen übertrifft. Pharmakologische und klinische Untersuchungen weisen auf verschiedene gesundheitsfördernde und therapeutische Eigenschaften hin oder haben diese schon dokumentiert. Weitere Abklärungen und Studien zur Wirksamkeit von Astaxanthin sind noch erforderlich. Die vorliegenden Resultate weisen jedoch darauf hin, dass die Substanz bald in der Medizin regelmässig als Medikament eingesetzt werden könnte.
5 Camera E, Mastrofrancesco A, Fabbri C, et al: Astaxanthin, canthaxanthin und beta-carotene differently affect UVA-induced oxidative damage and expression of oxidative responsive enzymes. Exp Dermatol 2009;18:222-231.

6 Yuan JP, Peng J, Yin K, et al: Potential healthpromoting effects of astaxanthin: a high-value carotenoid mostly from microalgae. Mol Nutr Food Res 2010;55:150-165.

7 Yamashita E: Eye fatigue (asthenopia) relief by astaxanthin. Anti-Aging Therapy 2009;11: 361-379.
8 Tominaga K, Hongo N, Karato M, et al: Cosmetic benefits of astaxanthin on human subjects. Acta Biochim Pol 2012;59:43-47.

9 Yamashita E: Astaxanthin and sports performance (in Japanese). Food Style 2011;15: 36-38.

10 Earnest CP, Lupo M, White KM, et al: Effect of astaxanthin on cycling time trial performance. Int J Sports Med 2011;32:882-888. 